\title{
ON LOCALLY ISOMETRIC MAPPINGS OF A $G$-SPACE ON ITSELF
}

\author{
W. A. KIRK
}

In his study [2] of locally isometric mappings of a $G$-space $\bar{R}$ on a $G$-space $R$, Busemann considers the following question: Under what conditions is every locally isometric mapping of a $G$-space $R$ on itself a motion? He proves that such is the case if either (i) the fundamental group of $R$ is not isomorphic to a proper subgroup of itself, or (ii) $R$ is compact. Busemann suggests $[2$, p. 405] that conditions (other than (i)) be sought which apply to noncompact spaces, in particular, conditions which apply to an ordinary cylinder. Szenthe replies to this in a recent paper [3] in which he finds conditions in terms of certain bounds on the lengths of nonoverlapping geodesic curves which begin and end at the same point. In [1] Busemann shows that under appropriate hypotheses on the order of magnitude of volumes of spheres a locally isometric mapping of a noncompact $G$-space on itself is a motion. Our paper provides another condition. We first show that if a locally isometric mapping of $R$ on itself has a fixed point, then it is a motion. From this it readily follows that if the motions of $R$ form a transitive group, then every locally isometric mapping of $R$ on itself is a motion.

Let $\phi$ denote a locally isometric mapping of a $G$-space $\bar{R}$ on a $G$ space $R$. The terminology we use and the following properties of $\phi$ are found in Busemann [2, \$27].

(1) If $\bar{x}(\tau), \alpha \leqq \tau \leqq \beta$, is a curve in $\bar{R}$ and if $\phi(\bar{x}(\tau))=x(\tau)$ represents $a$ segment, then $\bar{x}(\tau)$ represents a segment and $\bar{x}(\alpha) \bar{x}(\beta)=x(\alpha) x(\beta)$.

(2) For a given curve $x(\tau), \alpha \leqq \tau \leqq \beta$, in $R$ and a given point $\bar{a}$ of $\bar{R}$ such that $\phi(\bar{a})=x(\alpha)$ there is exactly one curve $\bar{x}(\tau)$ in $\bar{R}$ such that $\phi(\bar{x}(\tau))=x(\tau)$ with $\bar{x}(\alpha)=\bar{a}$.

(3) There is a number $\rho(p)>0$ such that if $\phi\left(p_{1}\right)=\phi\left(p_{2}\right)=p, p_{1} \neq p_{2}$, then $p_{1} p_{2} \geqq 2 \rho(p)$.

(4) The number of points of $\bar{R}$ which lie over a given point of $R$ is countable and is the same for different points of $R$.

(5) If $\phi$ is 1-1 then $\phi$ is an isometry.

Since each two points of a $G$-space are joined by a metric segment of the space, the following is an immediate consequence of (1) and (2).

Received by the editors March 4, 1963 and, in revised form, April 24, 1963. 
(6) If $a$ and $b$ are any two points of $R$ and if $\bar{a}$ is any point of $\bar{R}$ which lies over $a$, then there is a point $\bar{b}$ of $\bar{R}$ which lies over $b$, and $\bar{a} \bar{b}=a b$. It follows from the definition that

(7) If $\phi$ is a locally isometric mapping of $R$ on itself then $\phi^{n}$ is also $(n=1,2, \cdots)$.

TheOREM 1. If $\phi$ is a locally isometric mapping of a G-space $R$ on itself and if $\phi$ has a fixed point, then $\phi$ is a motion.

Proof. Suppose the contrary, and let $p$ denote any fixed point of $\phi$. By (5) $\phi$ is not $1-1$ so by (4) there is a point $p_{1} \neq p$ such that $\phi\left(p_{1}\right)=p$. By (6) there is a point $p_{2}$ such that $\phi\left(p_{2}\right)=p_{1}$ and $p p_{2}=p p_{1}$. We define inductively a sequence $\left\{p_{n}\right\}$ of points of $R$ such that $\phi\left(p_{n+1}\right)=p_{n}$ and $p p_{n}=p p_{1}, n=1,2, \cdots$.

If $n<m$ then $\phi^{n}\left(p_{n}\right)=p$ while $\phi^{n}\left(p_{m}\right)=p_{m-n}$. Since $p p_{m-n}=p p_{1}$, we see that $p_{m-n} \neq p$. Therefore $\phi^{n}\left(p_{n}\right) \neq \phi^{n}\left(p_{m}\right)$ and $p_{n} \neq p_{m}$. This shows that the elements of $\left\{p_{n}\right\}$ are pairwise distinct. By $(7) \phi^{n}$, for each positive integer $n$, is a locally isometric mapping of $R$ on itself, so by (3) $p_{i} p_{j} \geqq 2 \rho(p)$ if $i \neq j$. This contradicts the finite compactness of $R$.

TheOREM 2. A locally isometric mapping $\phi$ of a G-space $R$ on itself is a motion if and only if there is a motion $\psi$ of $R$ such that for some point $p \in R, \psi(\phi(p))=p$.

Proof. The necessity is trivial. The sufficiency is established by observing that $\psi \phi$ is a locally isometric mapping of $R$ on itself with fixed point $p$. By Theorem $1, \psi \phi$ is a motion and hence $1-1$. Therefore, $\phi$ is $1-1$ and by (5) a motion of $R$.

The motions of a $G$-space form a transitive group if, given any two points of the space, there is a motion of the space which maps one into the other. Thus the following theorem is a corollary to Theorem 2.

THEOREM 3. If a G-space $R$ has a transitive group of motions, then every locally isometric mapping of $R$ on itself is a motion.

Apparently little is known about the problem of determining the $G$-spaces with transitive groups of motion in general, but the 2dimensional case has been completely solved. Busemann has shown $[2$, p. 371] that the only $G$-surfaces (2-dimensional $G$-spaces) with transitive groups of motions are: The plane with a Minkowskian or quasi-hyperbolic metric, the cylinder and torus with a Minkowskian metric, the sphere and projective plane with a spherical metric.

The author is grateful to the referee for bringing reference [1] to his attention. 


\section{REFERENCES}

1. H. Busemann, Geometries in which the planes minimize area, Ann. Mat. Pura Appl. 55 (1961), 171-189.

2. - The geometry of geodesics, Academic Press, New York, 1955.

3. J. Szenthe, Über metrische Räume, deren lokalisometrische Abbildungen Isometrien sind, Acta Math. Acad. Sci. Hungar. 13 (1962), 433-441.

University of California, Riverside

\section{$A$-GENUS AND INDECOMPOSABILITY OF DIFFERENTIABLE MANIFOLDS}

\section{YASURÔ TOMONAGA}

Introduction. In the previous paper [1] we have studied the conditions on which a differentiable manifold be indecomposable and cited many examples of indecomposable manifolds. In this paper we shall study the relations between $A$-genus and indecomposability of a differentiable manifold.

1. Hereafter we denote by $X_{n}$ an $n$-dimensional compact orientable differentiable manifold. If $X_{n}=X_{r} \cdot X_{8}$ we say that $X_{n}$ is decomposable and if not, we say that $X_{n}$ is indecomposable. If $X_{4 n}=X_{n} \cdot X_{8}$ we have

$$
A\left(X_{4 n}\right)=A\left(X_{r}\right) A\left(X_{s}\right) ;
$$

where $A(X)$ denotes the $A$-genus of $X$ and we define as follows:

$$
A\left(X_{n}\right)=0, \quad n \not \equiv 0 \bmod 4 .
$$

If $r$ and $s$ are divisible by 4, the relation (1.1) follows from the general property of multiplicative series $[2$, p. 75]. According to the cobordism theory, the cobordism components of $X_{r}(r \neq 0$ mod 4$)$ consist only of torsions. Hence the product $X_{r} \cdot X_{s}$ also consists only of torsions. Therefore $A\left(X_{r} \cdot X_{s}\right)$ is zero. Thus (1.1) holds in general. Meanwhile Atiyah and Hirzebruch have proved the following:

Theorem 1 (Atiyah and Hirzebruch [3]). If $X_{4 n}$ is differentiably imbedded in the $(8 n-2 q)$-sphere, then $A\left(X_{4 n}\right)$ is divisible by $2^{q+1}$. If moreover $q \equiv 2 \bmod 4$, then $A\left(X_{4 n}\right)$ is divisible by $2^{q+2}$.

It is well known that an $X_{n}$ is always differentiably imbedded in the $2 n$-sphere. Hence we have from the above theorem

Received by the editors March 20,1963. 